\title{
REDES DE ATENÇÃO À SAÚDE COMO PRODUTORAS DE CUIDADO EM SAÚDE MENTAL: UMA ANÁLISE REFLEXIVA
}

| Carlos Bruno Silveira ${ }^{1}$; Lourdes Suelen Pontes Costa²; Maria Salete Bessa Jorge ${ }^{3}$ |

\section{RESUMO}

Objetivou-se analisar a produção científica brasileira acerca das redes de atenção à saúde (RAS) para o cuidado em saúde mental. Trata-se de um estudo bibliográfico com abordagem reflexiva acerca da temática do cuidado integral nas RAS no período de 2012 a 2016. Foi realizado um levantamento na biblioteca eletrônica Scielo, utilizando as palavras-chaves: Redes de Atenção à Saúde; Atenção Primária à Saúde e Atenção à Saúde Mental, sendo encontrados 51 artigos, que, após a leitura dos títulos e resumos, resultaram em 24 artigos para serem analisados. Mapeou-se quantitativamente a produção científica publicada acerca da RAS e foram identificados os significados e sentidos que constituem a produção selecionada. Utilizou-se da Análise Categorial Temática para inferência dos resultados, abordando as redes de cuidado com enfoque na integralidade, no processo de cuidado, reflexões, entraves e estratégias, além da formação dos profissionais de saúde com foco nas suas contribuições e seus desafios. As relações interligadas e preconizadas em redes de saúde ainda não foram consolidadas em sua totalidade, o que tem dificultado a concretização do cuidado integral à saúde, principalmente quando se refere à rede de saúde mental.

\section{PALAVRAS-CHAVE: Assistência à saúde; Atenção primária à saúde; Saúde mental}

\section{RESUMEN}

Redes de Atención a la Salud como productoras de cuidado en salud mental: Un análisis reflexiva”

Se objetivó analizar la producción científica brasileña acerca de las redes de atención a la salud para el cuidado en salud mental. Se trata de un estudio bibliográfico con abordaje reflexivo acerca de la temática del cuidado integral en las RAS en el período de 2012 a 2016. Se realizó un levantamiento en la biblioteca electrónica Scielo, utilizando las palabras clave: Redes de Atención a la Salud; La atención primaria a la Salud y Atención a la Salud Mental, se encontraron 51 artículos, que, después de la lectura de los títulos y resúmenes, resultaron en 24 artículos para ser analizados. Se mapeó cuantitativamente la producción científica publicada acerca de la RAS y se identificaron los significados y sentidos que constituyen la producción seleccionada. Se utilizó el análisis categorial temático para inferencia de los resultados, abordando las redes de cuidado con enfoque en la integralidad, en el proceso de cuidado, reflexiones, obstáculos y estrategias, además de la formación de los profesionales de salud con foco en sus contribuciones y en sus desafíos.Las relaciones interconectadas y preconizadas en redes de salud aún no han sido consolidadas en su totalidad, lo que ha dificultado la concreción del cuidado integral en salud, principalmente cuando se refiere a la red de salud mental.

DESCRIPTORES: Asistencia sanitaria; Atención primaria en salud; Salud mental

\begin{abstract}
"Health Care Networks as producers of mental health care: A reflective analysis"

The objective was to analyze the Brazilian scientific production about health care networks for mental health care. This is a bibliographical study with a reflexive approach on the theme of integral care in the RAS in the period from 2012 to 2016. A survey was carried out in the Scielo electronic library, using the following keywords: Health Care Networks; Primary Health Care and Mental Health Care, being found 51 articles, which, after reading the titles and abstracts, resulted in 24 articles to be analyzed. It is mapped quantitatively the scientific literature published about the RAS and the meanings have been identified and senses that constitute the selected production. For this purpose, the Thematic Categorical Analysis was used, addressing care networks with a focus on completeness, as well as the process of care, reflections, obstacles and strategies, as well as the training of health professionals with a focus on their contributions and challenges. The interconnected and recommended relationships in health networks have not yet been consolidated in their entirety, which has made it difficult to achieve comprehensive health care, especially when it refers to the mental health network.
\end{abstract}

KEYWORDS: Health care; Primary health care; Mental health

Submetido em 31-09-2017

Aceite em 14-02-2018

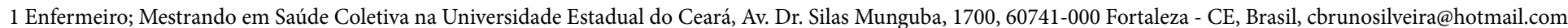

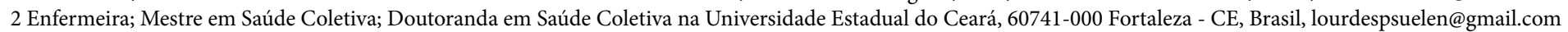

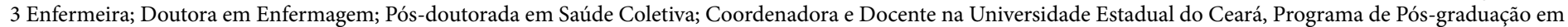
Saúde Coletiva, 60741-000 Fortaleza - CE, Brasil, maria.salete.jorge@gmail.com

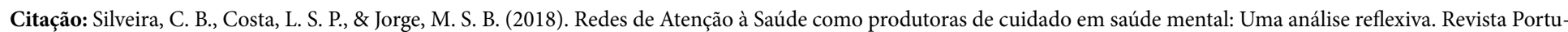
guesa de Enfermagem de Saúde Mental (19), 61-70. doi: 10.19131/rpesm.0203 


\section{INTRODUÇÃO}

A proposta de atenção integral à saúde no Brasil baseiase na construção de uma rede única de atenção à saúde, articulada em todas as suas instâncias, atendendo a população de forma regionalizada, integrada, hierarquizada e formada por diferentes dispositivos de atenção à saúde.

Nesse sentido, a Rede de Atenção à Saúde (RAS) emerge como proposta que deve conter serviços organizados em níveis de complexidade tecnológica, na qual se estabelecem suas ações e seus serviços, permitindo satisfazer as necessidades da pessoa no cuidado com a sua saúde, de modo a criar um itinerário sanitário racional e identitário, sem obstáculos burocráticos, com economia processual e temporal, racionalidade nos gastos e nos exames de apoio diagnóstico, entre outros elementos (Santos, 2017).

A coordenação da RAS pela Atenção Primária à Saúde(APS) pressupõe ser um processo complexo. Há um tensionamento de forças que se estabelecem no intra e nos interserviços de saúde, cujo vetor resultante poderá aproximar-se ou distanciar-se das ideias pensadas para o fortalecimento dessa proposta (Lapão, Arcenio, Popolin, e Rodrigues, 2017).

No âmbito da Saúde Mental, preconiza-se, mediante a implantação da Rede de Atenção Psicossocial (RAPS), ações e serviços que, mesmo sendo diversos e especializados em diferentes níveis de complexidade, se mantenham integrados por sistemas de apoio capazes de atender o sujeito em toda a sua singularidade, para além de partes isolada e fragmentadas como ocorre no modelo biomédico. Destaca-se a relevância da APS para a promoção de saúde nas comunidades, principalmente no desenvolvimento de ações que visem o rastreamento, encaminhamento e cuidado dos clientes que possuem necessidades de saúde mental, dado o impacto assistencial e financeiro que essas demandas trazem para a saúde pública (Organização Pan-americana da Saúde, 2001).

A incorporação do modelo psicossocial (orientado por serviços substitutivos de base territorial) ainda não está plenamente consolidada, pois existe oscilação entre as práticas de saúde orientadas pelo paradigma biomédico, cuja ações desenvolvidas são curativas e centradas no médico, em detrimento de outras necessidades individuais e coletivas que revelam a incorporação de um modelo ligado à proposta da saúde coletiva, que considera a prevenção e a promoção da saúde (Campos, Ferrer, Gama, Campos, Trapé e Dantas, 2014).
É incontestável o avanço promovido pelo modelo de Atenção Psicossocial, contudo, a consolidação desse modelo exige a transformação não apenas estrutural, mas também dos profissionais que são formados numa lógica positivista, centrado em um modelo biomédico, pautado em práticas curativas e medicamentosas o que dificulta sobremaneira a realização de uma assistência integral que privilegie a subjetividade da pessoa. Dessa forma, infere-se que o processo de construção desse cuidado requer constante revisão e atualização, por isso, diante dessas lacunas e aberturas, destaca-se a necessidade de uma nova estratégia de composição dessa rede de assistência (Acioli e Amarante, 2013).

Assim, Franco (2015) considera que o estudo da RAS se tornou numa das discussões mais desafiantes dos tempos atuais. Em primeiro lugar, pela atualidade, pois é prioridade em diversos países a organização dos serviços em redes e que estão em pleno processo de efetivação. Em segundo lugar, pela complexidade que o tema alcança, ao supormos que o trabalhador tem altos graus de liberdade no exercício do seu trabalho e, portanto, está apto a realizar as redes com altos graus de singularidade, diferenciando em muito do que é prescrito pelas normatizações oficiais.

Dito isto, surge à questão sobre como são discutidas as publicações científicas sobre o tema das redes de atenção à saúde para o cuidado em saúde mental no Brasil? Portanto, o estudo tem como objetivo analisar a produção científica brasileira acerca das redes de atenção à saúde para o cuidado em saúde mental.

\section{MÉTODOS}

Trata-se de um estudo bibliográfico com abordagem reflexiva acerca da temática do cuidado integral em saúde mental nas RAS entre os anos de 2012 a 2016 pois, nesse período tal questão ganha propulsão na efetivação da integralidade. Esse tipo de estudo envolve toda a bibliografia publicada sobre o tema em estudo, desde publicações avulsas a revistas, pesquisas e outros, propiciando a construção de um novo arcabouço sobre um determinado assunto e inovando com considerações críticas (Marconi e Lakatos, 2011).

Foi realizado um levantamento das bases de dados do campo da saúde, sendo definida a biblioteca eletrônica Scielo, que constitui uma biblioteca com amplo acesso a periódicos e artigos científicos e tem por objetivo o desenvolvimento de uma metodologia comum para a preparação, o armazenamento, a disseminação e a avaliação da produção científica em formato eletrônico. 
Para a seleção do material empírico deste estudo, utilizou-se os descritores cadastrados no DECS: "Atenção Primária à Saúde", "Atenção à Saúde Mental" e a palavra-chave "Rede de Atenção à Saúde". Optou-se por esta última, uma vez que não foi encontrado no DECS descritor que se assemelhasse às definições propostas anteriormente por Santos (2017) e Franco (2015).

Dessa forma, realizou-se o cruzamento dos termos supracitados através do operador booleano "AND". Para tanto utilizou-se como critérios de inclusão, artigos completos produzidos no Brasil, disponíveis na íntegra, publicados no período de 2012 a 2016 e nos idiomas português, inglês e espanhol. Os critérios de exclusão foram: artigos de revisão, teses, dissertações e editoriais. Destarte, inicialmente, foram encontrados 51 artigos que se aproximavam da temática em questão, contudo oito eram artigos repetidos, restando 43 dos quais realizou-se a leitura dos títulos e resumos, sendo ainda excluídos os artigos que não tratavam diretamente da temática proposta (redes de atenção à saúde e cuidado em saúde mental). Ao final, restaram 24 para serem analisados. Os mesmos serão apresentados de forma sintética no Quadro 1, a seguir.

Quadro 1 - Caracterização da Produção Científica acerca das Redes de Cuidados em Saúde, na APS e Saúde Mental

\begin{tabular}{|c|c|c|c|c|c|}
\hline No & Ano & Autores & Título & $\begin{array}{l}\text { Tipo de } \\
\text { Estudo/ } \\
\text { Metodo- } \\
\text { logia }\end{array}$ & Objetivo \\
\hline 1 & 2016 & $\begin{array}{l}\text { Paiva, } \\
\text { Torrenté, } \\
\text { Landim, } \\
\text { Branco, } \\
\text { Tamboril e } \\
\text { Cabral. }\end{array}$ & $\begin{array}{l}\text { Sofrimento } \\
\text { psíquico e a } \\
\text { abordagem da } \\
\text { comunidade na } \\
\text { voz do agente } \\
\text { comunitário de } \\
\text { saúde. }\end{array}$ & $\begin{array}{l}\text { Quali- } \\
\text { tativo/ } \\
\text { Pesquisa } \\
\text { de base } \\
\text { empírica } \\
\text { (Trans- } \\
\text { versal) }\end{array}$ & $\begin{array}{l}\text { Desvelar } \\
\text { modos de } \\
\text { perceber e } \\
\text { de lidar com } \\
\text { a doença em } \\
\text { contextos } \\
\text { específicos. }\end{array}$ \\
\hline 2 & 2016 & $\begin{array}{l}\text { Portugal, } \\
\text { Campos, } \\
\text { Correia, } \\
\text { Gonçalves, } \\
\text { Ballester, } \\
\text { Tófoli, } \\
\text { Mari, Gask, } \\
\text { Dowrick, } \\
\text { Bower e } \\
\text { Fortes. }\end{array}$ & $\begin{array}{l}\text { Rede de apoio } \\
\text { social, saúde } \\
\text { mental e quali- } \\
\text { dade de vida: } \\
\text { um estudo } \\
\text { transversal } \\
\text { na atenção } \\
\text { primária. }\end{array}$ & $\begin{array}{l}\text { Quanti- } \\
\text { tativo/ } \\
\text { Estudo } \\
\text { Transver- } \\
\text { sal }\end{array}$ & $\begin{array}{l}\text { Identificar a } \\
\text { associação } \\
\text { entre angús- } \\
\text { tia emocio- } \\
\text { nal e redes de } \\
\text { apoio social } \\
\text { na APS. }\end{array}$ \\
\hline 3 & 2015 & $\begin{array}{l}\text { Conceição, } \\
\text { Vicentin, } \\
\text { Leal, Ama- } \\
\text { ral, Fischer, } \\
\text { Kahhale, } \\
\text { Rosa, Spo- } \\
\text { laor e Saes. }\end{array}$ & $\begin{array}{l}\text { Interferên- } \\
\text { cias criativas } \\
\text { na relação } \\
\text { ensino-serviço: } \\
\text { itinerários de } \\
\text { um Programa } \\
\text { de Educação } \\
\text { pelo Trabalho } \\
\text { para a Saúde } \\
\text { (PET-Saúde). }\end{array}$ & $\begin{array}{l}\text { Quali- } \\
\text { tativo/ } \\
\text { Analítico }\end{array}$ & $\begin{array}{l}\text { Discutir a } \\
\text { integração } \\
\text { ensino- } \\
\text { serviço no } \\
\text { âmbito do } \\
\text { PET-Saúde } \\
\text { Mental. }\end{array}$ \\
\hline
\end{tabular}

\begin{tabular}{|c|c|c|c|c|c|}
\hline 4 & 2015 & $\begin{array}{l}\text { Rézio, } \\
\text { Moro, } \\
\text { Marcon e } \\
\text { Fortuna. }\end{array}$ & $\begin{array}{l}\text { Contribuições } \\
\text { do PET-Saúde/ } \\
\text { Redes de Aten- } \\
\text { ção Psicosso- } \\
\text { cial à Saúde da } \\
\text { Família }\end{array}$ & $\begin{array}{l}\text { Qualita- } \\
\text { tivo/ Ex- } \\
\text { ploratório } \\
\text { e Descri- } \\
\text { tivo }\end{array}$ & $\begin{array}{l}\text { Analisar o } \\
\text { Programa } \\
\text { de Educação } \\
\text { pelo Trabalho } \\
\text { para a Saúde } \\
\text { em uma uni- } \\
\text { dade de ESF. }\end{array}$ \\
\hline 5 & 2015 & $\begin{array}{l}\text { Teixeira, } \\
\text { Couto e } \\
\text { Delgado. }\end{array}$ & $\begin{array}{l}\text { Repercussões } \\
\text { do processo de } \\
\text { reestruturação } \\
\text { dos serviços de } \\
\text { saúde mental } \\
\text { para crianças } \\
\text { e adolescentes } \\
\text { na cidade de } \\
\text { Campinas, São } \\
\text { Paulo. }\end{array}$ & $\begin{array}{l}\text { Quantita- } \\
\text { tivo/ Ex- } \\
\text { ploratório- } \\
\text { descritivo- } \\
\text { documen- } \\
\text { tal }\end{array}$ & $\begin{array}{l}\text { Descrever e } \\
\text { analisar as } \\
\text { mudanças } \\
\text { na rede de } \\
\text { SMCA. }\end{array}$ \\
\hline 6 & 2015 & $\begin{array}{l}\text { Lima, } \\
\text { Soares, } \\
\text { Bilton, } \\
\text { Dias, } \\
\text { Ferrioll e } \\
\text { Perracini. }\end{array}$ & $\begin{array}{l}\text { Fatores re- } \\
\text { lacionados à } \\
\text { sonolência di- } \\
\text { urna excessiva } \\
\text { em idosos da } \\
\text { comunidade: } \\
\text { um estudo } \\
\text { exploratório. }\end{array}$ & $\begin{array}{l}\text { Quantita- } \\
\text { tivo/ Ex- } \\
\text { ploratório }\end{array}$ & $\begin{array}{l}\text { Investigar a } \\
\text { prevalência } \\
\text { de EDS e seus } \\
\text { correlatos } \\
\text { sociodemo- } \\
\text { gráficos, de } \\
\text { saúde física e } \\
\text { mental entre } \\
\text { idosos. }\end{array}$ \\
\hline 7 & 2015 & $\begin{array}{l}\text { Jorge, } \\
\text { Diniz, } \\
\text { Lima e } \\
\text { Penha. }\end{array}$ & $\begin{array}{l}\text { Apoio } \\
\text { matricial, pro- } \\
\text { jeto terapêu- } \\
\text { tico singular e } \\
\text { produção do } \\
\text { cuidado em } \\
\text { saúde mental. }\end{array}$ & $\begin{array}{l}\text { Qualita- } \\
\text { tivo }\end{array}$ & $\begin{array}{l}\text { Compreender } \\
\text { o apoio ma- } \\
\text { tricial, projeto } \\
\text { terapêutico } \\
\text { singular, e a } \\
\text { produção do } \\
\text { cuidado em } \\
\text { saúde mental. }\end{array}$ \\
\hline 8 & 2015 & Hirdes. & $\begin{array}{l}\text { A perspectiva } \\
\text { dos profission- } \\
\text { ais da Atenção } \\
\text { Primária à } \\
\text { Saúde sobre o } \\
\text { apoio matricial } \\
\text { em saúde } \\
\text { mental. }\end{array}$ & $\begin{array}{l}\text { Qualita- } \\
\text { tivo/ De- } \\
\text { scritivo- } \\
\text { analítico }\end{array}$ & $\begin{array}{l}\text { Investigar } \\
\text { o Apoio } \\
\text { Matricial em } \\
\text { saúde mental } \\
\text { na APS. }\end{array}$ \\
\hline 9 & 2015 & $\begin{array}{l}\text { Tszesn- } \\
\text { ioski, } \\
\text { Nóbrega, } \\
\text { Lima e } \\
\text { Fac- } \\
\text { undes. }\end{array}$ & $\begin{array}{l}\text { Constru- } \\
\text { indo a rede de } \\
\text { cuidados em } \\
\text { saúde mental } \\
\text { infantojuvenil: } \\
\text { intervenções } \\
\text { no território. }\end{array}$ & $\begin{array}{l}\text { Quali- } \\
\text { tativo/ } \\
\text { Pesquisa- } \\
\text { ação }\end{array}$ & $\begin{array}{l}\text { Descrever } \\
\text { a rede de } \\
\text { cuidados de } \\
\text { crianças em } \\
\text { sofrimento } \\
\text { psíquico. }\end{array}$ \\
\hline 10 & 2014 & $\begin{array}{l}\text { Costa, } \\
\text { Dimen- } \\
\text { stein e } \\
\text { Leite. }\end{array}$ & $\begin{array}{l}\text { Estratégias } \\
\text { de cuidado e } \\
\text { suporte em } \\
\text { saúde mental } \\
\text { entre mulheres } \\
\text { assentadas. }\end{array}$ & $\begin{array}{l}\text { Qualita- } \\
\text { tivo }\end{array}$ & $\begin{array}{l}\text { Investigar as } \\
\text { estratégias } \\
\text { de cuidado } \\
\text { e suporte } \\
\text { psicossocial à } \\
\text { mulheres. }\end{array}$ \\
\hline 11 & 2014 & $\begin{array}{l}\text { Fortes, } \\
\text { Menezes, } \\
\text { Athié, } \\
\text { Chazan, } \\
\text { Rocha, } \\
\text { Thiesen, } \\
\text { Ragoni, } \\
\text { Pithon e } \\
\text { Macha- } \\
\text { do. }\end{array}$ & $\begin{array}{l}\text { Psiquiatria no } \\
\text { século XXI: } \\
\text { transforma- } \\
\text { ções a partir } \\
\text { da integração } \\
\text { com a Atenção } \\
\text { Primária pelo } \\
\text { matriciamento. }\end{array}$ & $\begin{array}{l}\text { Qualita- } \\
\text { tivo }\end{array}$ & $\begin{array}{l}\text { Analisar } \\
\text { o papel da } \\
\text { Psiquiatria } \\
\text { nos sistemas } \\
\text { assistenciais } \\
\text { na APS. }\end{array}$ \\
\hline
\end{tabular}




\begin{tabular}{|c|c|c|c|c|c|}
\hline 12 & 2014 & $\begin{array}{l}\text { Hori e Nas- } \\
\text { cimento. }\end{array}$ & $\begin{array}{l}\text { O Projeto Ter- } \\
\text { apêutico Singu- } \\
\text { lar e as práticas } \\
\text { de saúde mental } \\
\text { nos Núcleos de } \\
\text { Apoio à Saúde } \\
\text { da Família } \\
\text { (NASF) em } \\
\text { Guarulhos (SP), } \\
\text { Brasil. }\end{array}$ & $\begin{array}{l}\text { Qualita- } \\
\text { tivo }\end{array}$ & $\begin{array}{l}\text { Analisar } \\
\text { as possi- } \\
\text { bilidades de } \\
\text { construção } \\
\text { de PTS pelas } \\
\text { equipes } \\
\text { de saúde } \\
\text { mental. }\end{array}$ \\
\hline 13 & 2014 & $\begin{array}{l}\text { Wetz- } \\
\text { ela, Pinho, } \\
\text { Olschowsky, } \\
\text { Guedes, } \\
\text { Camatta e } \\
\text { Schneider. }\end{array}$ & $\begin{array}{l}\text { A rede de } \\
\text { atenção à saúde } \\
\text { mental a partir } \\
\text { da Estraté- } \\
\text { gia Saúde da } \\
\text { Família. }\end{array}$ & $\begin{array}{l}\text { Quali- } \\
\text { tativo/ } \\
\text { Avaliativo }\end{array}$ & $\begin{array}{l}\text { Discutir a } \\
\text { RASM a } \\
\text { partir do } \\
\text { cotidiano de } \\
\text { uma ESF. }\end{array}$ \\
\hline 14 & 2014 & $\begin{array}{l}\text { Vello, Pop- } \\
\text { im, Carazzai } \\
\text { e Pereira. }\end{array}$ & $\begin{array}{l}\text { Saúde do Idoso: } \\
\text { percepções } \\
\text { relacionadas ao } \\
\text { atendimento. }\end{array}$ & $\begin{array}{l}\text { Qualita- } \\
\text { tivo }\end{array}$ & $\begin{array}{l}\text { Com- } \\
\text { preender } \\
\text { como o ido- } \\
\text { so percebe o } \\
\text { atendimento } \\
\text { na APS. }\end{array}$ \\
\hline 15 & 2014 & $\begin{array}{l}\text { Queiroz, } \\
\text { Gomes, } \\
\text { Reis, Knupp } \\
\text { e Aquino. }\end{array}$ & $\begin{array}{l}\text { Repertórios } \\
\text { interpretativos } \\
\text { de profission- } \\
\text { ais da rede de } \\
\text { saúde mental } \\
\text { e APS de Belo } \\
\text { Horizonte sobre } \\
\text { uso de drogas. }\end{array}$ & $\begin{array}{l}\text { Quantita- } \\
\text { tivo }\end{array}$ & $\begin{array}{l}\text { Levantar } \\
\text { concepções } \\
\text { sobre drogas } \\
\text { de profis- } \\
\text { sionais de } \\
\text { saúde men- } \\
\text { tal\APS. }\end{array}$ \\
\hline 16 & 2014 & $\begin{array}{l}\text { Drummond, } \\
\text { Radicchi e } \\
\text { Gontijo. }\end{array}$ & $\begin{array}{l}\text { Fatores sociais } \\
\text { associados a } \\
\text { transtornos } \\
\text { mentais com } \\
\text { situações de } \\
\text { risco na atenção } \\
\text { primária de } \\
\text { saúde. }\end{array}$ & $\begin{array}{l}\text { Quanti- } \\
\text { tativo/ } \\
\text { Transver- } \\
\text { sal }\end{array}$ & $\begin{array}{l}\text { Avaliar } \\
\text { portadores } \\
\text { de transtor- } \\
\text { nos mentais } \\
\text { atendidos } \\
\text { nas unidades } \\
\text { de APS. }\end{array}$ \\
\hline 17 & 2013 & $\begin{array}{l}\text { Moliner e } \\
\text { Lopes. }\end{array}$ & $\begin{array}{l}\text { Saúde mental } \\
\text { na atenção } \\
\text { básica- pos- } \\
\text { sibilidades para } \\
\text { uma prática } \\
\text { voltada para } \\
\text { a ampliação e } \\
\text { integralidade da } \\
\text { saúde mental. }\end{array}$ & $\begin{array}{l}\text { Qualita- } \\
\text { tivo }\end{array}$ & $\begin{array}{l}\text { Conhecer } \\
\text { como vem } \\
\text { se con- } \\
\text { figurando as } \\
\text { práticas em } \\
\text { saúde mental } \\
\text { na APS. }\end{array}$ \\
\hline 18 & 2013 & $\begin{array}{l}\text { Jorge, Sousa } \\
\text { e Franco. }\end{array}$ & $\begin{array}{l}\text { Apoio matri- } \\
\text { cial- dispositivo } \\
\text { para resolução } \\
\text { de casos clíni- } \\
\text { cos de saúde } \\
\text { mental na At- } \\
\text { enção Primária } \\
\text { à Saúde. }\end{array}$ & $\begin{array}{l}\text { Quali- } \\
\text { tativo/ } \\
\text { Estudo de } \\
\text { Caso }\end{array}$ & $\begin{array}{l}\text { Com- } \\
\text { preender a } \\
\text { constituição } \\
\text { do apoio } \\
\text { matricial no } \\
\text { âmbito da } \\
\text { APS. }\end{array}$ \\
\hline 19 & 2013 & $\begin{array}{l}\text { Paes, } \\
\text { Schimith, } \\
\text { Barbosa e } \\
\text { Righi. }\end{array}$ & $\begin{array}{l}\text { Rede de aten- } \\
\text { ção em saúde } \\
\text { mental na } \\
\text { perspectiva dos } \\
\text { coordenadores } \\
\text { de serviços de } \\
\text { saúde. }\end{array}$ & $\begin{array}{l}\text { Quali- } \\
\text { tativo/ } \\
\text { Descri- } \\
\text { tivo-ex- } \\
\text { ploratório }\end{array}$ & $\begin{array}{l}\text { Com- } \\
\text { preender } \\
\text { como coor- } \\
\text { denadores } \\
\text { de serviços } \\
\text { de saúde } \\
\text { percebem a } \\
\text { RASM. }\end{array}$ \\
\hline
\end{tabular}

\begin{tabular}{|c|c|c|c|c|c|}
\hline 20 & 2012 & $\begin{array}{l}\text { Moura, } \\
\text { Guedes, } \\
\text { Freire, Bes- } \\
\text { sa, Braga, e } \\
\text { Silva. }\end{array}$ & $\begin{array}{l}\text { Planejamento } \\
\text { familiar de } \\
\text { mulheres com } \\
\text { transtorno } \\
\text { mental- o que } \\
\text { profissionais } \\
\text { do CAPS têm a } \\
\text { dizer. }\end{array}$ & $\begin{array}{l}\text { Qualitati- } \\
\text { vo/ Estudo } \\
\text { de campo }\end{array}$ & $\begin{array}{l}\text { Verificar } \\
\text { demandas de } \\
\text { planejamen- } \\
\text { to familiar } \\
\text { que chegam } \\
\text { ao CAPS. }\end{array}$ \\
\hline 21 & 2012 & $\begin{array}{l}\text { Minozzo, } \\
\text { Kammzets- } \\
\text { er, Debas- } \\
\text { tiani, Fait e } \\
\text { Paulon. }\end{array}$ & $\begin{array}{l}\text { Grupos de } \\
\text { saúde mental } \\
\text { na atenção } \\
\text { primária à } \\
\text { saúde. }\end{array}$ & $\begin{array}{l}\text { Quali- } \\
\text { tativo/ } \\
\text { Pesquisa - } \\
\text { Interven- } \\
\text { ção }\end{array}$ & $\begin{array}{l}\text { Analisar } \\
\text { as práticas } \\
\text { de cuidado } \\
\text { nos grupos } \\
\text { de saúde } \\
\text { mental. }\end{array}$ \\
\hline 22 & 2012 & $\begin{array}{l}\text { Pini e Waid- } \\
\text { man. }\end{array}$ & $\begin{array}{l}\text { Fatores inter- } \\
\text { ferentes nas } \\
\text { ações da equipe } \\
\text { da Estratégia } \\
\text { Saúde da Famí- } \\
\text { lia ao portador } \\
\text { de transtorno } \\
\text { mental }\end{array}$ & $\begin{array}{l}\text { Qualita- } \\
\text { tivo/ Ex- } \\
\text { ploratório- } \\
\text { descritivo }\end{array}$ & $\begin{array}{l}\text { Conhecer os } \\
\text { fatores que } \\
\text { interferem } \\
\text { nas ações ao } \\
\text { portador de } \\
\text { transtorno } \\
\text { mental. }\end{array}$ \\
\hline 23 & 2012 & $\begin{array}{l}\text { Almeida, } \\
\text { Nunes, } \\
\text { Camey, } \\
\text { Pinheiro e } \\
\text { Schmidt. }\end{array}$ & $\begin{array}{l}\text { Transtornos } \\
\text { mentais em } \\
\text { uma amostra } \\
\text { de gestantes da } \\
\text { rede de atenção } \\
\text { básica de saúde } \\
\text { no Sul do } \\
\text { Brasil. }\end{array}$ & $\begin{array}{l}\text { Estudo de } \\
\text { coorte }\end{array}$ & $\begin{array}{l}\text { Estimar a } \\
\text { prevalência } \\
\text { de transtor- } \\
\text { nos mentais } \\
\text { na gestação. }\end{array}$ \\
\hline 24 & 2012 & $\begin{array}{l}\text { Chiavagatti, } \\
\text { Kantorski, } \\
\text { Willrich, } \\
\text { Cortes, } \\
\text { Jardim e } \\
\text { Rodrigues. }\end{array}$ & $\begin{array}{l}\text { Articulação } \\
\text { entre Centros } \\
\text { de Atenção } \\
\text { Psicossocial } \\
\text { e Serviços de } \\
\text { Atenção Básica } \\
\text { de Saúde }\end{array}$ & $\begin{array}{l}\text { Quali- } \\
\text { tativo/ } \\
\text { descritivo }\end{array}$ & $\begin{array}{l}\text { Analisar as } \\
\text { formas de } \\
\text { articulação } \\
\text { entre o } \\
\text { CAPS e a } \\
\text { APS. }\end{array}$ \\
\hline
\end{tabular}

Posteriormente, fez-se a leitura exaustiva dos textos, através da síntese de cada artigo e da delimitação do eixo temático correspondente. Os dados foram analisados através da articulação de abordagens quantitativa e qualitativa. Mapeou-se quantitativamente a produção científica publicada acerca da RAS, referentes a ano de publicação, tipo de produção, centro de estudo, área do conhecimento, periódicos, local do estudo, abordagem, objetivos, técnicas de análise, síntese dos resultados e eixos temáticos.

Para a fase qualitativa, buscou-se entender os significados e sentidos que formam a produção relacionada com o tema da pesquisa na base de dados escolhida. Trabalhou-se, portanto, com a Análise Categorial Temática proposta por Minayo (2010) e retraduzida por Assis e Jorge (2010).

Extraíram-se, então, das produções analisadas, possíveis convergências, divergências e complementaridades, seguindo a ordem das fases: pré-análise; exploração do material; categorização; tratamento dos resultados obtidos e interpretação (Assis e Jorge, 2010; Minayo, 2010). 


\section{RESULTADOS E DISCUSSÕES}

Por meio da análise dos artigos coletados, emergiram três categorias temáticas que serão o mote das discussões apresentadas a seguir, sendo estas: Redes de cuidado na atenção psicossocial com enfoque na integralidade; Processo de cuidado em saúde mental: reflexões, entraves e estratégias para a integralidade; Formação dos profissionais de saúde para atuar em saúde mental: contribuições e desafios. Pode-se perceber na literatura que houve momentos de oscilações nas publicações científicas sobre a temática das RAS, revelando um decréscimo no último ano, 2016.

O Gráfico 1 representa a distribuição dos estudos selecionados e inclui os trabalhos publicados no período de 2012 a 2016.

Gráfico 1 - Distribuição absoluta da publicação científica no período de 2012 a 2016

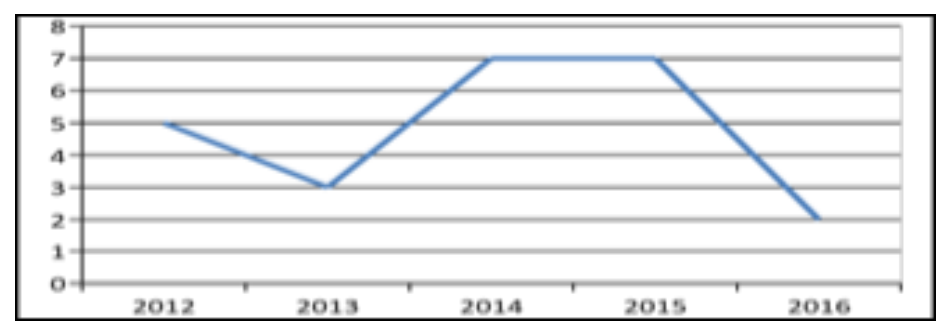

Concernente à região onde os estudos foram desenvolvidos, identificou-se maior concentração nas Regiões Sudeste e Sul do Brasil, sendo encontrados nove e oito artigos respectivamente. Destes, destacam-se os estados de São Paulo e Rio Grande do Sul, com seis publicações cada. A Região Nordeste aparece com seis publicações e o estado do Ceará concentrando a maior parte dos estudos encontrados, quatro. A Região Centro-Oeste aparece com dois estudos e a Região Norte com nenhuma publicação. Em um dos trabalhos encontrados, a pesquisa foi realizada em duas cidades de regiões diferentes simultaneamente, no caso, Sudeste e Centro-Oeste.

Os estudos encontrados foram desenvolvidos em vinte centros de pesquisas, dos quais dezoito são oriundos de universidades públicas, sendo doze federais e seis estaduais, e cinco universidades de iniciativa privada. Esses números demonstram que a maioria das pesquisas é desenvolvida em universidades públicas, revelando que, apesar do contexto de crise política vigente, são delas que partem os maiores investimentos. A iniciativa privada ainda investe pouco no desenvolvimento de pesquisas nesse âmbito.

Quando analisadas as áreas de conhecimento, constatou-se que a categoria que mais se destacou foi a Enfermagem, com nove publicações.
A Psicologia aparece com três publicações e a Medicina com duas. Dez das publicações foram consideradas como de categoria multiprofissional, uma vez que pelo menos um dos autores possuía formação acadêmica diferente dos demais.

Nesse sentido, considera-se os periódicos que publicaram os trabalhos selecionados como referências para as estratégias de cuidado integral, ganhando destaque as seguintes revistas apresentadas no Quadro 2.

Quadro 2 - Lista de Periódicos e Quantidade de Artigos Encontrados em Cada Periódico no Período de 2012 a 2016

\begin{tabular}{|l|c|}
\hline Periódico & Quantidade \\
\hline Acta Paul Enferm & 1 \\
\hline Cad. Saúde Pública & 2 \\
\hline Ciência \& Saúde Coletiva & 3 \\
\hline Esc Anna Nery & 1 \\
\hline Estudos de Psicologia & 1 \\
\hline Fractal, Rev. Psicol & 1 \\
\hline Interface - Comunicação Saúde Educação & 2 \\
\hline Physis Revista de Saúde Coletiva & 1 \\
\hline Rev Bras Enferm & 1 \\
\hline Rev Bras Epidemiol & 2 \\
\hline Rev Esc Enferm USP & 3 \\
\hline Rev Gaúcha Enferm & 1 \\
\hline Revista Colombiana de psicologia & 1 \\
\hline Saúde Debate & 1 \\
\hline Saúde Soc. & 1 \\
\hline Texto Contexto Enferm & 1 \\
\hline Trab. Educ. Saúde & 1 \\
\hline
\end{tabular}

Quanto à abordagem dos estudos, 18 artigos pesquisados apresentam-se como de abordagem qualitativa e seis de abordagem quantitativa. Os tipos de estudos evidenciados na investigação são variados, porém os descritivos prevalecem quando comparados aos outros, no entanto os demais apresentam abordagens próprias de estudos qualitativos, como estudos de caso, pesquisaação, avaliação e outros, como exposto no Gráfico 2.

Gráfico 2 - Tipos de estudos referentes aos artigos de 2012 a 2016

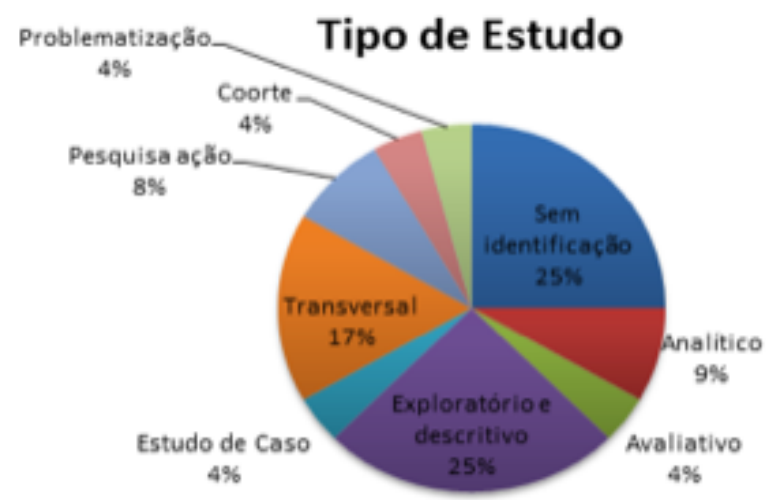


Quanto às técnicas de análise, encontrou-se que a mais utilizada foi a proposta por Bardin - Análise de Conteúdo (7), em seguida a Análise Temática - Minayo (4) e aparecem ainda Análise Documental (3), Hermenêutica e Dialética (2), Metodologia da Problematização (2), Análise Inferencial (1), Avaliação de Quarta Geração (1) e Avaliações por Escalas Quantitativas (4).

No que diz respeito às características dos artigos publicados no período entre 2012 e 2016 sobre a temática da RAS como produtora de cuidado no âmbito da saúde mental, destaca-se que os estados do Sul e Sudeste do País alavancam as pesquisas, sendo estas em sua maioria de abordagem qualitativa, demonstrando a importância da natureza desses estudos.

No que se refere à análise qualitativa, emergiram três categorias temáticas de acordo com os temas mais discutidos nos artigos analisados: a primeira trata especificamente das redes de cuidado e de suas especificidades, com ênfase na integralidade e na atenção psicossocial; a segunda já se refere principalmente às estratégias utilizadas para se conseguir fazer com que o cuidado em saúde mental aconteça em rede; e a terceira traz a formação do trabalhador como essencial à construção desse modelo de atenção.

$\mathrm{Na}$ primeira categoria, intitulada "Redes de cuidado na atenção psicossocial brasileira: enfoque na integralidade", foram identificados artigos que destacaram a necessidade de estreitar as relações das ações entre Atenção Primária à Saúde e Saúde Mental, devendo estas se constituírem como estratégias fundamentais para a consolidação da rede, considerando a dinamicidade desse processo de construção.

Identifica-se que é essencial ao Sistema Único de Saúde (SUS) unir os entes federativos em uma determinada região para delimitar o seu campo de atuação e organizar as referências de serviços dadas suas complexidades sanitárias, tecnológicas e financeiras. Sem esse formato organizacional, não se consegue viabilizar um sistema integral, universal e igualitário (Santos, 2017).

As práticas clínicas de cuidado relacionadas ao adoecimento psíquico ainda se expressam em uma tecnologia de biopolítica de gestão, entendida como medicalização social, que se mostra difundida e aceita na contemporaneidade. As ações priorizam o diagnóstico orgânico, as terapias medicalizantes e, principalmente, medicamentalizantes, as quais perfazem caminhos unidirecionais em que, ao sujeito, não restam muitas escolhas além de adaptarem-se ao processo de cuidado oferecido (Bezerra, Morais, Paula, Silva e Jorge, 2016).
Com isso, ressalta-se que as ações de saúde ainda são desenvolvidas com foco no atendimento às condições agudas (não minimizando sua importância), contudo torna-se primordial descortinar esse cenário e trazer à cena os demais elementos necessários para a garantia da integralidade.

Outro entrave que vale destacar é que há um consenso entre os profissionais relatando que a relação de referência/contrarreferência realizada com a especialidade de saúde mental e demais carece de melhoria. Alguns médicos foram até mais enfáticos na crítica a esse modelo (Campos e Amarante, 2015).

Uma alternativa seria as Redes de Referenciação que regulam as relações de complementaridade e de apoio técnico entre as entidades intervirem por meio de um prontuário eletrônico, de forma a promoverem o acesso dos clientes aos cuidados e serviços de que efetivamente necessitam, bem como sustentarem o sistema integrado de informação interinstitucional (Lapão et al., 2017). Identifica-se que a articulação da assistência em saúde mental, por meio de uma rede interativa e articulada, constitui desafio no atual cenário inconstante das políticas públicas de saúde no Brasil. Corrobora-se também a prerrogativa de que a APS tem papel fundamental na promoção da qualidade de vida de pessoas que vivem com algum sofrimento psíquico, cabendo a esta ordenar a rede de cuidados, acolhendo e orientando sempre que necessário.

A segunda categoria refere-se ao processo de cuidado em saúde mental no Brasil: reflexões, entraves e estratégias para a integralidade. Nesta, as principais concepções debatidas nos artigos versam acerca da produção de arranjos organizacionais do sistema de saúde, de modo a diminuir a fragmentação dos processos de trabalho e valorizar o cuidado multi e interdisciplinar, considerando os clientes nos seus contextos, para que possa ser construído um projeto terapêutico adequado, singular e contínuo.

Porém, os artigos destacam que o processo saúde-doença mental atende ainda predominantemente ao modelo clínico individual, organicista e biológico na maneira de conceber o transtorno mental, contrapondo-se ao modelo de atenção psicossocial, bem como à atual política de organização dos serviços de saúde na perspectiva da Reforma Psiquiátrica (Campos e Amarante, 2015).

$\mathrm{Na}$ maioria das vezes, os clientes descrevem o acesso ao serviço da APS como algo burocrático e demorado, desde o tempo de espera do agendamento até o dia da consulta. Atribuem essas dificuldades à falta de profissionais médicos e à alta rotatividade destes. 
O acolhimento é apontado como um espaço de escuta aos seus problemas e de orientação, mas o criticam por constituir-se em uma forma de controlar o acesso àquilo que é mais esperado: a consulta médica (Campos et al., 2014).

A rotatividade dos profissionais é tema constante nas discussões sobre APS, agravada pela precariedade dos vínculos trabalhistas e pela ausência de uma política para gestão do trabalho. Por conseguinte, todos os esforços empreendidos para capacitação e educação permanente que visam melhorar o processo de trabalho estão sempre recomeçando, comprometendo a longitudinalidade do cuidado (Almeida, Santos, Santos e Silveira, 2016).

Com isso, destaca-se que a corresponsabilização faz-se elemento relevante na relação entre as pessoas quando se estabelece o cuidado, quer seja de medicamentos, quer seja de relações interpessoais entre o cuidador e o sujeito que é cuidado. Na concepção do cuidado em que há predominância do modelo centrado no médico, os sujeitos não possuem o poder de decidir sobre seu tratamento, o que implica em ausência de autonomia (Bezerra et al., 2016).

É possível que a mudança de compreensão por parte da população, a respeito da importância das diversas "clínicas" na área da saúde e da necessidade do estabelecimento de fluxos e regulações, possa ocorrer por meio de uma atividade pedagógica desenvolvida pela própria equipe.

Percebe-se a necessidade de trocas e diálogos entre os profissionais - que são portadores de saberes técnicocientíficos - e a população, de modo que possa ser construído de forma compartilhada um conceito sobre o processo saúde-doença (Campos et al., 2014).

Os estudos analisados também apontam o Apoio Matricial como importante articulador da rede de serviços de saúde, aumentando o leque de possibilidades e a circulação dos clientes nos diversos equipamentos do sistema de saúde, com destaque para os serviços de atenção primária. Assim, o apoio matricial entre equipes pode ser apreendido como uma metodologia de trabalho que garante a retaguarda especializada, tanto em nível assistencial como técnico-pedagógico. Com isso, o CAPS (Centro de Atenção Psicossocial) que possui a função de serviço especializado, não estaria desresponsabilizando-se pelo cuidado, mas passaria a atuar a partir da descentralizar esse cuidado, levando-o para mais próximo do usuário (Chiavagatti, Kantorski, Willrich, Cortes, Jardim, e Rodrigues, 2012).
No entanto, apesar de ter sido reconhecido de maneira unânime como ferramenta essencial à efetivação do cuidado em saúde mental, o Apoio Matricial ainda tem enfrentado múltiplos entraves. Dentre eles, destacam-se a insuficiente adesão dos profissionais às atividades, a falta de conhecimentos específicos em saúde mental, a rotatividade dos profissionais nos serviços e a falta de envolvimento das gestões dos serviços e do município no processo de planejamento e execução das atividades (Tatmatsu e Araújo, 2016).

Além destes, foram identificados os aspectos relacionados às noções de interdisciplinaridade; a relação de longitudinalidade que propicia o vínculo; o tempo para a escuta; o sigilo; o respeito; a não dissociação de saúde física e mental; a sensibilidade para identificar o sofrimento; as necessidades próprias da pessoa; as rupturas nas formas de cuidado nos grupos de saúde mental na APS; a forma de ajuda; e o interesse de ambos em qualificar a atenção em saúde mental e diminuir o sofrimento. Dessa forma, o trabalho de Tszesnioski, Nóbrega, Lima e Facundes (2015) reitera a necessidade de práticas intersetoriais para a promoção da independência e autonomia dos sujeitos.

Entende-se que novos arranjos e esforços deveriam ser efetivados com a finalidade de reestruturar o processo de trabalho das equipes na APS e dos núcleos de apoio e de aumentar a capacidade de dar resposta às necessidades de saúde integral da população.

É imprescindível que os processos de trabalho produzam cuidado e para tal se faz necessária a utilização de tecnologias leves, dos afetos, dos vínculos e da corresponsabilização a fim de estimular o compromisso de trabalhadores, clientes e familiares com a produção do cuidado.

$\mathrm{Na}$ terceira categoria, emergiu o tema da formação dos profissionais de saúde brasileiros para atuar em saúde mental: contribuições e desafios, destacando a necessidade de intervenções a nível macro através de políticas governamentais destinadas a favorecer a prática assistencial interdisciplinar para garantir a integralidade e assim subsidiar melhorias nas condições de saúde.

Faz-se de suma importância que o profissional da APS se integre com a comunidade para compreender o seu papel psicossocial e espiritual, buscando interpretar a realidade vivida por cada integrante, seja individual ou coletivamente, para que possa direcioná-lo para uma vida saudável através do empoderamento e assim, fomentar ações de promoção em saúde (Campos e Amarante, 2015). 
A capacidade de comunicação entre os integrantes de uma equipe é fundamental ao seu bom funcionamento, o que se reverte em melhoria da qualidade dos serviços de saúde e da capacidade de adaptação. O processo formativo de um clínico passa por aprender e contextualizar conhecimentos com os que sabem através da ação, o que leva paulatinamente a um maior aperfeiçoamento (Lapão et al., 2017).

Parte-se, então, de uma concepção de cuidado que implica a atuação dentro dos espaços de convivência dos clientes, por isso, torna-se importante a criação de novos circuitos de socialização para que se construa uma rede que funcione com base na participação social em diversas áreas.

Assim, são necessários equipamentos intersetoriais, espaços em que diferentes setores possam contribuir para o cuidado e o desenvolvimento da autonomia do indivíduo (Acioli e Amarante, 2013).

Para atuar na APS e realizar um atendimento tecnicamente consistente, é necessária uma formação que amplie o campo de percepção clínica, sob o risco de se desenvolver uma atuação profissional superficial, ineficiente e de baixa resolutividade (Campos e Amarante, 2015).

Um mecanismo inovador foi a instituição do plano de carreira aos trabalhadores vinculados à APS em Portugal, contexto que difere do Brasil, acarretando prejuízos como a dificuldade de fixação de profissionais de saúde, somada à desmotivação, ao cumprimento parcial de carga-horária, à falta de conhecimento sobre os atributos da APS e a uma visão retrógrada baseada em atividades programáticas sobre a organização dos serviços (Lapão et al., 2017).

São necessárias intervenções a nível macro, através de políticas governamentais destinadas à formação dos profissionais, a fim de reduzir essas diferenças e construir uma saúde com mais qualidade.

Considera-se, pois, que ações diretas no nível dos serviços da ponta podem gerar grandes avanços para a saúde da população. Coloca-se, ainda, a função que as instituições fomentadoras das políticas de saúde devem exercer como provedoras da formação dos profissionais de saúde.

\section{CONSIDERAÇÕES FINAIS}

As relações interligadas e preconizadas em redes de saúde ainda são incipientes e insuficientes para a concretização do cuidado integral em saúde, principalmente quando se refere à rede de saúde mental. Para que a assistência não fique restrita aos eventos agudos, precarizando o enfoque preventivo e promotor de saúde, algo tão importante, porém pouco desenvolvido no contexto da atenção psicossocial no cenário brasileiro, assim reitera-se a importância da interligação tanto no nível micro como macro. Esse processo deve perpassar desde a prática da interdisciplinaridade durante o desenvolvimento dos processos de trabalho até o âmbito da corresponsabilização dos serviços em seus diversos níveis de complexidade, destacando os serviços substitutivos com suas diversas características necessárias para assistência aos sujeitos em sofrimento mental.

A APS, que deveria atuar como coordenadora do cuidado no interior das RAS, ainda não consegue exerce tal função com plenitude, fazendo com que as ações de cuidado não sejam prestadas de forma contínua. Assim, reitera-se a necessidade de fortalecimento da APS para o fortalecimento da temática das RAS, bem como para a garantia da integralidade do cuidado, ou seja, uma assistência contínua aos indivíduos com ênfase nas suas necessidades e particularidades, não sendo restrita ao adoecimento mental, mas considerando todas as dimensões que constituem o sujeito.

\section{REFERÊNCIAS BIBLIOGRÁFICAS}

Acioli, M. L., e Amarante, P. D. C. (2013). O acompanhamento terapêutico como estratégia de cuidado na atenção psicossocial. Psicologia: Ciência e Profissão, 33(4), 964-975.

Almeida, M. S., Nunes, M. A., Camey, S., Pinheiro, A. P., e Schmidt, M. A. (2012). Transtornos mentais em uma amostra de gestantes da rede de atenção básica de saúde no Sul do Brasil. Cadernos de Saúde Pública, 28(2), 385393. doi: 10.1590/S0102-311X2012000200017

Almeida, P. F., Santos, A. M., Santos, V. P., e Silveira Filho, R. M. (2016). Integração assistencial em região de saúde: Paradoxo entre necessidades regionais e interesses locais. Saúde e Sociedade, 25(2), 320-335. doi: 10.1590/S0104-12902016153295 
Assis, M. M., e Jorge, M. S. B. (2010). Métodos de análise em pesquisa qualitativa. In J. S. S. Santana, e M. A. A. Nascimento (Eds.), Pesquisa: Métodos e técnicas de conhecimento da realidade social (pp. 147-155). Feira de Santana: UEFS Editora.

Bezerra, I. C., Morais, J. B., Paula, M. L., Silva, T. M. R., e Jorge, M. S. B. (2016). Uso de psicofármacos na atenção psicossocial: Uma análise à luz da gestão do cuidado. Saúde em Debate, 40(110), 148-161. doi: 10.1590/01031104201611011

Campos, R. T. O., Ferrer, A. L., Gama, C. A. P., Campos, G. W. S., Trapé, T. L., e Dantas, D. V. (2014). Avaliação da qualidade do acesso na atenção primária de uma grande cidade brasileira na perspectiva dos usuários. Saúde em Debate, 38(n.spe), 252-264. doi: 10.5935/01031104.2014S019

Campos, A., e Amarante, P. D. C. (2015). Estudo sobre práticas de cuidado em saúde mental na Atenção Primária: O caso de um município do interior do estado do Rio de Janeiro. Cadernos Saúde Coletiva, 23(4), 425435. doi: 10.1590/1414-462X201500040226

Chiavagatti, F. G., Kantorski, L. P., Willrich, J. Q., Cortes, J. M., Jardim, V. M. R., e Rodrigues, C. G. S. S. (2012). Articulação entre Centros de Atenção Psicossocial e Serviços de Atenção Básica de Saúde. Acta Paulista de Enfermagem, 25(1), 11-17. doi: 10.1590/S010321002012000100003

Conceição, M. R., Vicentin, M. C. G., Leal, B. M. M. L., Amaral, M. M., Fischer, A. B., Kahhale, E. M. P., ...e Saes, D. (2015). Interferências criativas na relação ensino-serviço: Itinerários de um Programa de Educação pelo Trabalho para a Saúde (PET-Saúde). Comunicação Saúde e Educação, 19(Supl I), 745-755. doi: 10.1590/180757622014.0894

Costa, M. G., Dimenstein, M., e Leite, J. (2015). Estratégias de cuidado e suporte em saúde mental entre mulheres assentadas. Revista Colombiana de Psicología, 24(1), 13-28. doi: 10.15446/rcp.v24n1.41968

Drummond, B. L. C., Radicchi, A. L. A., e Gontijo, E. C. D. (2014). Fatores sociais associados a transtornos mentais com situações de risco na atenção primária de saúde. Revista Brasileira de Epidemiologia, 17(Supl. 2), 68-80. doi: 10.1590/1809-4503201400060006
Franco, T. B. (2015). Redes de cuidado: Conexão e fluxos para o bom encontro com a saúde. In P. F. Almeida, A. M. Santos, e M. K. B. Souza (Eds.), Atenção primária à saúde na coordenação do cuidado em regiões de saúde (pp. 261-276). Salvador: EDUFBA.

Fortes, S. F., Menezes, A., Athié, K., Chazan, L. F., Rocha, H., Thiesen, J., ...e Machado, A. (2014). Psiquiatria no século XXI: Transformações a partir da integração com a Atenção Primária pelo matriciamento. Revista de Saúde Coletiva, 24(4), 1079-1102. doi: 10.1590/S010373312014000400006

Hirdes, A. (2015). A perspectiva dos profissionais da Atenção Primária à Saúde sobre o apoio matricial em saúde mental. Ciência \& Saúde Coletiva, 20(2), 371-382. doi: 10.1590/1413-81232015202.11122014

Hori, A. A., e Nascimento, A. F. (2014). O Projeto Terapêutico Singular e as práticas de saúde mental nos Núcleos de Apoio à Saúde da Família (NASF) em Guarulhos (SP), Brasil. Ciência \& Saúde Coletiva, 19(8), 3561-3571. doi: 10.1590/1413-81232014198.11412013

Jorge, M. S. B., Diniz, A. M., Lima, L. L., e Penha, J. C. (2015). Apoio matricial, projeto terapêutico singular e produção do cuidado em saúde mental. Texto \& Contexto - Enfermagem, 24(1), 112-120. doi: 10.1590/010407072015002430013

Jorge, M. S. B., Sousa, F. S. P., e Franco, T. B. (2013). Apoio matricial - dispositivo para resolução de casos clínicos de saúde mental na Atenção Primária à Saúde. Revista Brasileira de Enfermagem, 66(5), 738-744.

Lapão, L. V., Arcencio, R. A., Popolin, M. P., e Rodrigues, L. B. (2017). Atenção Primária à Saúde na coordenação das Redes de Atenção à Saúde no Rio de Janeiro, Brasil, e na região de Lisboa, Portugal. Revista Ciência \& Saúde Coletiva, 22(3), 713-724. doi: 10.1590/141381232017223.33532016

Lima, C. A., Soares, W. J. S., Bilton, T. L., Dias, R. C., Ferrioll, E., e Perracini, M. R. (2015). Fatores relacionados à sonolência diurna excessiva em idosos da comunidade: Um estudo exploratório. Revista Brasileira de Epidemiologia, 18(3), 607-617. doi: 10.1590/19805497201500030007 
Marconi, M. A., e Lakatos, E. M. (2011). Metodologia do trabalho científico: Procedimentos básicos, pesquisa bibliográfica, projeto e relatório, publicações e trabalhos científicos. São Paulo: Atlas.

Minayo, M. C. S. (2010). Pesquisa social: Teoria, método e criatividade. Petrópolis: Vozes.

Minozzo, F., Kammzetser, C. S., Debastiani, C., Fait, C. S., e Paulon, S. M. (2012). Grupos de saúde mental na atenção primária à saúde. Fractal: Revista de Psicologia, 24(2), 323-340.

Moliner, J., e Lopes, S. M. B. (2013). Saúde mental na atenção básica - possibilidades para uma prática voltada para a ampliação e integralidade da saúde mental. Saúde e Sociedade, 22(4), 1072-1083.

Moura, E. R. F., Guedes, T. G., Freire, S. A., Bessa, A. T., Braga, V. A., e Silva, R. M. (2012). Planejamento familiar de mulheres com transtorno mental - o que profissionais do CAPS têm a dizer. Revista da Escola de Enfermagem da USP, 46(4), 935-943.

Organização Pan-americana da Saúde. Organização Mundial da Saúde. (2001). Relatório sobre a saúde no mundo 2001: Saúde mental: Nova concepção, nova esperança. Genebra: OPAS/OMS.

Paes, L. G., Schimith, M. D., Barbosa, T. M., e Righi, L. B. (2013). Rede de atenção em saúde mental na perspectiva dos coordenadores de serviços de saúde. Trabalho, Educação e Saúde, 11(2), 395-409. doi: 10.1590/S198177462013000200008

Paiva, P. C., Torrenté, M. O N., Landim, F. L. P., Branco, J. G. O., Tamboril, B. C. R., e Cabral, A. L.T. (2016). Sofrimento psíquico e a abordagem da comunidade na voz do agente comunitário de saúde. Revista da Escola de Enfermagem, 50(n. esp.), 139-144. doi: 10.1590/S0080623420160000300020

Pini, J. S., e Waidman, M. A. P. (2012). Fatores interferentes nas ações da equipe da Estratégia Saúde da Família ao portador de transtorno mental. Revista da Escola de Enfermagem da USP, 46(2), 372-379. doi: 10.1590/ S0080-62342012000200015
Portugal, F. B., Campos, M. R., Correia, C. R., Gonçalves, D. A., Ballester, D., Tófoli, L. F., ...e Fortes, S. (2016). Rede de apoio social, saúde mental e qualidade de vida: Um estudo transversal na atenção primária. Cadernos de Saúde Pública, 32(12), 1-11.

Queiroz, I. S., Gomes, A. P., Reis, A. L., Knupp, D. F. D., e Aquino, C. R. (2014). Repertórios interpretativos de profissionais da rede de saúde mental e APS de Belo Horizonte sobre uso de drogas. Saúde em Debate, 38(100), 80-93. doi: 10.5935/0103-104.20140005

Rézio, L. A., Moro, T. N, Marcon, S. R., e Fortuna, C. M. (2015). Contribuições do PET-Saúde/Redes de Atenção Psicossocial à Saúde da Família. Comunicação Saúde e Educação, 19(Supl I), 793-803. doi: 10.1590/180757622014.0673

Santos, L. (2017). Região de saúde e suas redes de atenção: Modelo organizativo-sistêmico do SUS. Revista Ciência \& Saúde Coletiva, 22(4), 1281-1289. doi: 10.1590/1413-81232017224.26392016

Tatmatsu, D. B., e Araújo, A. C. C. (2016). Atenção primária e saúde mental: Contribuições e potencialidades do apoio matricial. Mudanças em Psicologia da Saúde, 24(2), 71-79.

Teixeira, M. R., Couto, M. C. V., e Delgado, P. G. G. (2015). Repercussões do processo de reestruturação dos serviços de saúde mental para crianças e adolescentes na cidade de Campinas, São Paulo. Estudos de Psicologia, 32(4), 695-703. doi: 10.1590/0103-166X2015000400012

Tszesnioski, L. C., Nóbrega, K. B. G., Lima, M. L. T., e Facundes, V. L. D. (2015). Construindo a rede de cuidados em saúde mental infantojuvenil: Intervenções no território. Ciência \& Saúde Coletiva, 20(2), 363-370.

Vello, L. S., Popim, R. C., Carazzai, E. M., e Pereira, M. A. O. (2014). Saúde do Idoso: Percepções relacionadas ao atendimento. Escola Anna Nery, 18(2), 330-335. doi: $10.5935 / 1414-8145.20140048$

Wetzel, C., Pinho, L. B., Olschowsky, A., Guedes, A. C., Camatta, M. W., e Schneider, J. F. (2014). A rede de atenção à saúde mental a partir da Estratégia Saúde da Família. Revista Gaúcha de Enfermagem, 35(2), 27-32. doi: 10.1590/1983-1447.2014.02.43052 